\title{
„Ja, und jetzt lässt du das 'в' weg und setzt das 'футбол' in den Instrumental.“' Eine empirische Untersuchung zur mündlichen Fehlerkorrektur im Russischunterricht
}

The present article deals with the correction of oral grammatical mistakes in Russian lessons, which were recorded and coded quantitatively and qualitatively with the help of a detailed observation sheet during the lessons of a 11th grade Russian course in a comprehensive school. The teacher's approach to verbal student errors was examined with regard to the type of feedback chosen in relation to the focus of the lesson activity, the addressee, and the nature of the feedback signal, and taking into account the teacher's affective response. The evaluation of the obtained data showed that the use of correction types with negative evidence slightly outweighed in the observed study group, but the choice of feedback type especially depended on the parameters of activity focus and the expected degree of familiarity of the grammatical form. Errors that occurred in content-related phases and those related to grammatical rules with low familiarity were mostly corrected by model-delivering strategies. In contrast, errors in form-focused sequences and those related to known rules were mostly countered by elicitation. The corrections were mainly verbally addressed to the students who produced errors and in only a few cases were associated with affective positive feedback.

Статья посвящена проблеме исправления устных грамматических ошибок на уроке русского языка. В ходе исследования проводилась запись наблюдений за исправлениями учителя во время уроков русского языка в одиннадцатом классе общеобразовательной школы с учетом как количественных, так и качественных аспектов. 
Особенности исправления устных ошибок учеников были исследованы с точки зрения видов комментария, выбранных учителем в зависимости от фокуса деятельности на уроке, адресата и сигнала обратной связи. Также была учтена аффективная реакция учителя. Анализ собранных данных показал следующее: несмотря на то, что в исследуемой группе учеников преобладало непрямое исправление ошибок, выбор вида обратной связи зависел, прежде всего, от параметров фокуса деятельности и ожидаемой степени изученности грамматической формы. Ошибки, связанные с пониманием содержания или относящиеся к еще неизвестным ученикам правилам грамматики, чаще всего исправлялись с помощью моделей и образцов правильных ответов. В свою очередь, ошибки во время работы над грамматикой и ошибки, которые относились к знакомым правилам, чаще всего были исправлены напрямую, с указанием правильной формы. Исправления были адресованы ученикам преимущественно в устной форме и только в редких случаях были связаны с прямым исправлением ошибки, сопровождающимся эмоциональной реакцией.

\section{Einleitung}

Die Bedeutung des Fehlers im und für den Fremdsprachenunterricht hat sich in den vergangenen Jahrzehnten stark verändert. Der negativ konnotierte Fehler ist im Zusammenhang mit der Interlanguage-Hypothese einer Auffassung des Fehlers als einem Signal für interne Lernprozesse (vgl. Tönshoff 2005: 3) und demnach einer positiv konnotierten Lernchance gewichen. Damit aber auch SchülerInnen den Fehler als eine solche Chance zur Verbesserung ihrer Sprachkenntnisse begreifen und ihre Angst, Fehler zu machen, verlieren, ist der Umgang der Lehrkraft mit sprachlichen Normabweichungen und ihre grundsätzliche Einstellung zum Fehler im 
Fremdsprachenunterricht von gravierender Bedeutung. Welche Fehlerkultur in der Schule tatsächlich gepflegt wird, kann nicht nur von Schule zu Schule, sondern von Lehrkraft zu Lehrkraft und selbst von Lerngruppe zu Lerngruppe variieren und stimmt nicht zwangsläufig mit den Empfehlungen der Fremdspracherwerbsforschung überein (vgl. Stadler 2014: 282).

Damit einhergehend hat sich auch die Rolle der Fehlerkorrektur verändert (vgl. Schoormann \& Schlak 2011: 45 ff), die den SchülerInnen eine Gelegenheit zur punktuellen Verbesserung ihrer Sprachkenntnisse signalisiert. Denn in den meisten Fällen sind SchülerInnen auf eine Rückmeldung hinsichtlich ihrer Äußerungen angewiesen, um aus ihren Fehlern zu lernen (vgl. Stadler 2014: 282). Dieses Feedback erfolgt im Fremdsprachenunterricht überwiegend durch die Lehrkraft. Die Thematisierung von Fehlern im Unterrichtsgespräch veranlasst die SchülerInnen also nicht nur dazu, diese zu bemerken, sondern sie auch zu verbessern und dadurch ihr Sprachniveau anzuheben.

Die Entwicklung kommunikativer Kompetenz, die in den bildungsministeriellen Vorgaben Nordrhein-Westfalens besonders betont wird, gilt als primäres Ziel des schulischen Fremdsprachenunterrichts. Dadurch richtet sich der Blick vor allem auf die Korrektur mündlicher Fehler, die nachweislich die Aufmerksamkeit der LernerInnen lenkt und somit den Erwerb der Fremdsprache fördern kann (vgl. Tönshoff 2005: 16). Auch im Kernlehrplan für den Russischunterricht werden die interkulturelle sowie funktionale kommunikative Kompetenz weit in den Vordergrund gerückt (vgl. KLP 2014: 18). Zudem verfügt das Russische als stark flektierende Sprache vor allem im grammatischen Bereich über ein hohes Fehlerpotential (vgl. Stadler 2014: 282), das zahlreiche Korrekturen erforderlich macht.

Die Betrachtung und Untersuchung der mündlichen Fehlerkorrektur im Russischunterricht erfährt aber nicht nur vor dem Hintergrund dieser Rahmenbedingungen ihre Legitimation. Sie ist auch aus theoretischer Perspek-

Anastasia Drackert und Katrin Bente Karl (Hg.), Didaktik der slawischen Sprachen

Beiträge zum 2. Arbeitskreis in Innsbruck (19.02.-20.02.2018)

(C) 2019 innsbruck university press, ISBN 978-3-903187-80-1, DOI 10.15203/3187-80-1 
tive durchaus relevant, da bisher kaum empirische Untersuchungen in diesem Unterrichtsfach durchgeführt wurden. Diese sind grundsätzlich wichtig für die Weiterentwicklung sprachspezifischer Unterrichtsverfahren, Vermittlungsansätze und somit eines erfolgreichen Russischunterrichts, an dem SchülerInnen mit Freude teilnehmen. Ziel der Untersuchung ist es daher, einen kleinen Beitrag zur Erforschung dieses Faches zu leisten. Mithilfe eingehender Unterrichtsbeobachtung wurden am Beispiel einer Lehrkraft der Umgang mit mündlichen Fehlern und ihrer Korrektur im Russischunterricht analysiert.

\section{Mündliche Fehlerkorrektur - ein Überblick über Theorie und Forschung}

Die Erforschung der mündlichen Fehlerkorrektur, die in den Bereich der Fremdsprachenforschung gehört, erfolgt seit über 30 Jahren und erfreut sich eines steigenden Interesses. Das Forschungsfeld gestaltet sich allerdings als dermaßen breit und komplex, dass die empirische Wissenschaft bisher keine eindeutigen und v.a. für die Praxis anwendbaren Ergebnisse vorlegen konnte. Ein Blick auf die Forschungsgeschichte verrät allerdings einen enormen gedanklichen Fortschritt: Die ursprüngliche Skepsis gegenüber negativem Feedback ${ }^{1}$ beim L2-Erwerb konnte mit der Zeit auf der Grundlage kognitiv-interaktionistischer Ansätze überwunden und durch die Akzeptanz der positiven Wirkung von Feedback abgelöst werden. Die empirische Untersuchung von Feedback führte schließlich zu zahlreichen Klassifizierungsversuchen verschiedener Feedbacktypen und mündete in der Erforschung der Effektivität mündlicher Fehlerkorrektur (vgl. Schoormann \& Schlak 2011: 44), aus deren Ergebnissen immer wieder versucht

${ }^{1}$ Negatives Feedback, als Rückmeldung der Lehrkraft auf eine fehlerhafte Schüleräußerung, wird in dieser Arbeit synonym zum Begriff Fehlerkorrektur verwendet.

Anastasia Drackert und Katrin Bente Karl (Hg.), Didaktik der slawischen Sprachen

Beiträge zum 2. Arbeitskreis in Innsbruck (19.02.-20.02.2018)

() 2019 innsbruck university press, ISBN 978-3-903187-80-1, DOI 10.15203/3187-80-1 
wird, Korrekturempfehlungen für Lehrkräfte abzuleiten. Diese positive Entwicklung der Haltung gegenüber negativem Feedback beim L2-Output und der Akzeptanz positiver Auswirkungen mündlicher Fehlerkorrektur geht einher mit der Veränderung der Bewertung des Fehlers im Fremdsprachenunterricht an sich. Seitdem der Fehler als Indikator für Lernfortschritt aufgefasst wird, d.h. als ein Beleg dafür, dass LernerInnen Hypothesen über die L2 aufstellen und diese bei der Sprachproduktion auf ihre Richtigkeit testen, übernimmt die Fehlerkorrektur in Form von Feedback zu eben diesen Hypothesen die entscheidende Funktion, den Lernprozess voranzutreiben (vgl. ebd.: 47). Dass sich korrektives Feedback positiv auf den Erwerb der L2 auswirkt und diesen fördert, konnte mittlerweile auch durch diverse Studien nachgewiesen werden (vgl. ebd. 62; Tönshoff 2005: 16). Darüber, in welcher Form korrektives Feedback dem Lernenden beim L2-Erwerb am besten helfen kann, streitet sich die Forschung allerdings noch bis heute ${ }^{2}$, da die Effektivität der Korrektur von vielerlei lernerinternen sowie -externen Faktoren beeinflusst werden kann (vgl. Schoormann \& Schlak 2011: $63 \mathrm{ff})$.

Zu den zentralen Fragen der Erforschung zur Fehlerkorrektur gehören solche nach der Art und dem Zeitpunkt der Korrektur, der Instanz, die den mündlichen Fehler korrigiert, sowie nach der Entscheidung für oder gegen eine Korrektur. Die Arten oder Strategien der mündlichen Fehlerkorrektur werden in der einschlägigen Literatur meist nach der von Lyster \& Ranta (1997) vorgeschlagenen und als maßgebend zu bezeichnenden Taxonomie klassifiziert. Dabei wird zwischen den folgenden sechs Feedbackarten unterschieden:

1. explizite Korrekturen

2. recasts

\footnotetext{
${ }^{2}$ Vgl. hierzu die beiden gegensätzlichen Positionen von Long (1996, 2007), der implizite Feedbackstrategien bevorzugt, und Lyster (1998a, 2002a, 2002b, 2007), der sich für die größeren Lerneffekte bei explizitem Feedback ausspricht (vgl. Schoormann \& Schlak 2011: 57).
}

Anastasia Drackert und Katrin Bente Karl (Hg.), Didaktik der slawischen Sprachen

Beiträge zum 2. Arbeitskreis in Innsbruck (19.02.-20.02.2018)

(C) 2019 innsbruck university press, ISBN 978-3-903187-80-1, DOI 10.15203/3187-80-1 
3. Klärungsaufforderung

4. Fehlerwiederholung

5. metalinguistisches Feedback

6. Elizitierung

Bei den ersten beiden Feedbackarten, die im Folgenden unter dem Sammelbegriff Korrekturtypen I zusammengefasst werden, handelt es sich um solche mit einer sog. positiven Evidenz. D.h. dass dem Lerner bei der Korrektur ein zielsprachliches Modell durch die Lehrkraft bzw. InteraktionspartnerIn geboten wird. Während explizite Korrekturen (1) ausdrücklich auf den gemachten Fehler verweisen und diesen korrigieren (z.B. „Nein, nicht 'gehte', sondern 'ging'.") ${ }^{3}$, wird durch recasts (2) nur implizit angedeutet, dass ein Fehler gemacht wurde, indem die fehlerhafte Äußerung in korrigierter Form von der Lehrkraft wiederholt wird (z.B. S: „Dann gehte er nach Hause.“ - L: „Dann ging er nach Hause.“). Auch bei Klärungsaufforderungen (3) (z.B. „Wie bitte?") und Fehlerwiederholungen (4), bei denen die fehlerhafte Äußerung mit ggf. besonderer Betonung von der Lehrkraft wiederholt wird, handelt es sich um implizite Feedbackarten, die dem Lernenden in indirekter Weise mitteilen, dass er einen Fehler gemacht hat. Kommentare, Informationen oder Fragen bezüglich der fehlerhaften Äußerung (z.B. „Sagt man 'gehte' auf Deutsch?“, „Ist das maskulin?“) werden als metalinguistisches Feedback (5) bezeichnet. Elizitierungen (6) umfassen Aufforderungen an den Lernenden die fehlerhafte Äußerung selbstständig umzuformulieren. Dabei bittet der/die LehrerIn den/die SchülerIn in direkter Weise den Fehler zu verbessern oder lässt ihm Zeit einen Satz bzw. ein Wort zu vervollständigen, dessen Fehlerort er durch einen Abbruch beim Sprechen kennzeichnet (z.B. „Das ist ein...?“). Im Unterschied zu expliziten Korrekturen und recasts bieten die letzten vier Feedbackstrategien dem Lernenden keine zielsprachlichen Modelle, sondern ausschließlich negative Evidenz. Im Rahmen dieser Untersuchung werden sie als Korrekturtyp II klassifiziert. Diese Feedbackstrategien werden i.d.R.

\footnotetext{
${ }^{3}$ Beispiele entnommen aus Schoormann \& Schlak (2011).
} 
unter dem Terminus prompts zusammengefasst, da sie den Lernenden auffordern, seinen Fehler selbst zu korrigieren. (vgl. Schoormann \& Schlak 2011: $50 \mathrm{f}$ )

In Abhängigkeit vom Feedbacktyp kann die Korrekturinstanz, d.h. derjenige, der die Korrekturhandlung ausübt, variieren. Dies kann bei Feedbackstrategien mit positiver Evidenz (Korrekturtyp I) logischerweise nur die Lehrkraft bzw. InteraktionspartnerIn sein. Im Falle von prompts (Korrekturtyp II) hingegen werden die Lernenden zu einer Selbstkorrektur (SK) aufgefordert. Das Feedback kann dabei entweder an den Lernenden adressiert sein, der den Fehler gemacht hat und nun zu einer Eigenkorrektur (EK) aufgefordert wird. Oder aber es richtet sich an einen Mitlernenden, weshalb es sich dann um eine Fremdkorrektur (FK) handelt (vgl. Kleppin 1997: 86 ff). Ferner kann zwischen verschiedenen AdressantInnen differenziert werden: die Korrekturinitiierung kann beispielsweise durch den/die LehrerIn oder aber eine/n MitschülerIn erfolgen. Bemerkt der Urheber des Fehlers seinen Irrtum selbst, kann er ihn entweder eigenständig beheben oder seine Unsicherheit in Bezug auf seine Äußerung ausdrücken und dadurch ebenfalls eine Korrektur initiieren. Auch der Korrekturzeitpunkt kann zwischen einer sofortigen (beiläufigen) Korrektur, einer Korrektur am Ende der Lerneräußerung sowie einer eigenständigen Korrekturphase, die z.T. auch eine ganze Unterrichtseinheit in Anspruch nehmen kann, variieren (vgl. ebd. 107).

Die Art und Weise der Fehlerkorrektur kann aber auch nach weiteren Kriterien, wie der Aufforderungsart und der affektiven Beschaffenheit des Feedbacks, unterschieden werden. Die Aufforderung zur Selbstkorrektur kann dem jeweiligen Adressaten sowohl verbal als auch nonverbal, z.B. durch Mimik, Gestik oder zuvor in der Lerngruppe vereinbarte Zeichen signalisiert werden (vgl. Kleppin 1997: 96 ff). Außerdem kann die Lehrkraft affektiv unterschiedlich auf Fehler reagieren und den SchülerInnen dadurch eine positive oder aber negative Botschaft auf sozialer Ebene vermitteln, womit sie einen nicht zu vernachlässigenden Einfluss auf die 
Lernermotivation ausüben kann (vgl. ebd. 110 f).

Um die genannten Kernfragen nach der Form des Feedbacks, den initiierenden Parteien und ihrer Art, die Notwendigkeit zur Korrektur zu signalisieren, zu beantworten, werden von der Feedbackforschung Untersuchungen mit unterschiedlichen Ansätzen durchgeführt. Grob lassen sich Untersuchungen zur Fehlerkorrektur in zwei Gruppen einteilen: eine deskriptiver und eine entwicklungsbezogener Natur. Deskriptiv orientierte Studien unternehmen den Versuch, anhand von genauer Unterrichtsbeobachtung unterstützt durch Audio- oder Videoaufzeichnungen mündliche Fehlerkorrektur detailliert zu beschreiben und zu analysieren (z.B. Ellis, Basturkmen \& Loewen 2001; Kim \& Han 2007). ${ }^{4}$ Dabei können unterschiedliche Aspekte im Fokus der Untersuchungen stehen: die Erfassung der Struktur von Korrektursequenzen und die Beschreibung des kommunikativen Lehrerbzw. Lernerverhaltens (z.B. Fagan 2015), Klassifikation und Quantifizierung verschiedener Feedbacktypen (z.B. Kleppin \& Königs 1991), Identifizierung von individuellen Korrekturstilen (z.B. Kleppin \& Königs 1991; Lochtman 2002; Nystrom 1983), die lehrer- sowie lernerseitige affektive Einstellung zur Fehlerkorrektur (z.B. Cathcart \& Olsen 1976; Demme 2001; Gnutzmann \& Kiffe 1993; Havranek 2002; Kleppin \& Königs 1991; Schulz 2001 u.a.) etc. (vgl. Tönshoff 2005: 4 f). Solche Untersuchungen haben durch ihre beschreibende Natur zwar auf den ersten Blick keinen direkten praktischen Wert für den Unterrichtsalltag, können jedoch dabei helfen, „Fremdsprachenlehrer [...] für die Korrekturproblematik zu sensibilisieren und [...] [ihnen] in systematischer Weise Handlungsalternativen aufzuzeigen“ (ebd.: 8). Entwicklungsbezogene Studien zielen hingegen meist auf die Messung der relativen Effektivität von Feedback ab. ${ }^{5}$ Solche Studien messen vor dem

\footnotetext{
${ }^{4}$ Für eine umfangreiche Literaturliste siehe Schoormann \& Schlak (2011).

${ }^{5}$ Die Effektivität von mündlichen Fehlerkorrekturen lässt sich aber auch durchaus mithilfe deskriptiver Untersuchungen feststellen, indem beispielsweise sog. uptakes, d.h.
} 
Hintergrund bestimmter Erwerbshypothesen kurz-, mittel- und langfristige Erwerbsfortschritte in der L2 (vgl. ebd.: 8 f). Hierzu werden meist Prätests, eine Behandlungsphase und anschließende Posttests zur Überprüfung des Wirkungsgrades verschiedener Arten von Fehlerkorrektur herangezogen (vgl. Schoormann \& Schlak 2011: 53). Im Unterschied zu deskriptiven Studien werden hier neben quasi-experimentellen Datenerhebungsmethoden (Unterrichtsstudien in bestehenden Fremdsprachenklassen) auch experimentelle Designs herangezogen, bei denen Daten in einer laborähnlichen Situation außerhalb des Unterrichts erhoben werden, um die Anzahl der Störvariablen zu minimieren (vgl. Tönshoff 2005: 13). Die Ergebnisse der Ermittlung von Feedbackeffektivität schwanken aber nicht nur aufgrund der unterschiedlichen Untersuchungsdesigns, sondern unterliegen v.a. dem Einfluss zahlreicher lernerexterner und -interner Faktoren (vgl. Schoormann \& Schlak 2011: $63)$.

Trotz zahlreicher Studien im Bereich der mündlichen Fehlerkorrektur gibt es nur wenige, die sich mit der Reaktion von LehrerInnen auf mündliche Schülerfehler und dem Umgang mit diesen im Unterrichtsverlauf beschäftigen, obwohl gerade solche Untersuchungen auch praktische Hinweise für Lehrkräfte liefern könnten (vgl. Fagan 2015: 74 f). Ausnahme und gleichzeitig Vorbild für die vorliegende Untersuchung bildet daher die auf der Konversationsanalyse basierende Studie von Fagan (2015), in der u.a. die affektive Reaktion der Lehrperson auf fehlerhafte Schüleräußerungen beleuchtet wird. ${ }^{6}$ Fagan untersucht den Zusammenhang zwischen der Struktur der Fehlerkorrektur des Lehrers/der Lehrerin und ihrer Auswirkung auf die Lerngelegenheit für die SchülerInnen in authentischen institutionalisierten Lernsituationen. Er berücksichtigt dabei nicht nur das verbale und

„verschiedene Arten von Reaktionen der Lernenden auf korrektives Feedback“ (Schoormann \& Schlak 2011: 55) beobachtet und analysiert werden.

${ }^{6}$ Eine Beschreibung der affektiven Dimension von Korrekturepisoden liefert außerdem die Arbeit von Kleppin \& Königs (1991).

Anastasia Drackert und Katrin Bente Karl (Hg.), Didaktik der slawischen Sprachen

Beiträge zum 2. Arbeitskreis in Innsbruck (19.02.-20.02.2018)

(C) 2019 innsbruck university press, ISBN 978-3-903187-80-1, DOI 10.15203/3187-80-1 
nonverbale Verhalten der Lehrkraft und den Korrekturtyp, sondern analysiert auch, welchem Muster die Erteilung eines positiven bzw. negativen Feedbacks folgt und an wen es in Abhängigkeit davon adressiert ist. Als Methode wählt Fagan eine Konversationsanalyse auf Mikroebene und konzentriert sich auf den dritten Turn der Lehrkraft in einer sog. 'InitiationRespond-Feedback'-Sequenz, die er auf der Grundlage aufgezeichneter Unterrichtsgespräche durchführt. Er stellt zwei verschiedene Verhaltensweisen der Lehrperson fest, die sich nach dem Umfang des Fehlers richten, wobei in beiden Fällen vor der eigentlichen Fehlerkorrektur ein positives Feedback erfolgt. Bei Schüleräußerungen, die teilweise richtig sind, wird zunächst der Erfolg bzw. die Leistung auf einer persönlichen Ebene hervorgehoben, bevor die Lehrkraft eine Korrekturaufforderung äußert, die sich jedoch an die MitschülerInnen richtet. Dieses Vorgehen wird aber nur bei Aufgaben angewendet, bei denen es um sprachliche Richtigkeit geht. Auch für komplett falsche Äußerungen erhalten die LernerInnen zunächst ein positives Feedback, das eine persönliche Wertschätzung der Kreativität der Antwort seitens der Lehrkraft ausdrückt. Das negative Feedback ist auch hier auf die Mitlernenden und nicht denjenigen ausgerichtet, der den Fehler gemacht hat (vgl. Fagan 2015: 82). Das beobachtete und analysierte Korrekturverhalten betont nicht die Wissenslücken der LernerInnen, sondern das, was sie leisten können. Es fördert dadurch ihre Motivation und bestärkt sie darin, sich in der Fremdsprache auszuprobieren. Diese Studie zeigt in erster Linie, dass es lohnenswert ist, auch über eine reine Klassifikation von Feedback hinaus die konkrete kommunikative Interaktion und die Ausrichtung von negativem Feedback genau zu beobachten, um differenziert über positive oder negative Effekte sprechen zu können. 
Für das Russische als Fremdsprache existieren grundsätzlich nur einige wenige empirische Untersuchungen ${ }^{7}$ und bisher keine Studien im Bereich der mündlichen Fehlerkorrektur. Um diesem Forschungsdesiderat entgegenzuwirken, unternimmt diese Studie den Versuch, mündlich vorgebrachtes negatives Feedback im Russischunterricht einer primär quantitativen Untersuchung mit qualitativen Ausläufen im Bereich der affektiven Lehrerreaktionen zu unterziehen.

\section{Untersuchungsgegenstand und Forschungsfragen}

Diese Untersuchung unterliegt einem deskriptiven Ansatz und widmet sich der Beobachtung des Lehrerverhaltens beim Auftreten von mündlichen Grammatikfehlern im Russischunterricht. Es soll untersucht werden, wie Lehrkräfte fehlerhaften Schüleräußerungen auf unterschiedlichen Ebenen in Echtzeit begegnen.

Dafür soll zunächst beobachtet werden, welchen Korrekturtyp die Lehrkraft bevorzugt verwendet, d.h. ob sie sich eher für den Korrekturtyp I mit positiver Evidenz (explizite Korrektur, recasts) entscheidet oder aber den Korrekturtyp II mit negativer Evidenz (prompts) vorzieht, und das prozentuale Verhältnis dieser beiden Korrekturtypen ermittelt werden. Im Vergleich zum Anfängerunterricht, in dem die Korrektur meist direkt durch den/die LehrerIn erfolgt (vgl. Kleppin 1997: 89) und daher überwiegend als Typ I zu klassifizieren wäre, ist v.a. in fortgeschrittenen Lerngruppen mit einer überwiegenden Anwendung von Korrekturtyp II zu rechnen, da die SchülerInnen bereits über ein umfangreicheres Vorwissen in der russischen Sprache und Grammatik verfügen, auf das sie bei einer vom/von der

${ }^{7}$ Für einen Überblick zu empirischer Forschung innerhalb der Fachdidaktik des Russischen in Deutschland siehe Mehlhorn (2019).

Anastasia Drackert und Katrin Bente Karl (Hg.), Didaktik der slawischen Sprachen

Beiträge zum 2. Arbeitskreis in Innsbruck (19.02.-20.02.2018)

(C) 2019 innsbruck university press, ISBN 978-3-903187-80-1, DOI 10.15203/3187-80-1 
LehrerIn initiierten Selbstkorrektur zurückgreifen können. Allerdings sind in solchen Gruppen grundsätzlich weniger Fehlerkorrekturen insgesamt und v.a. solche im grammatischen Bereich zu erwarten (vgl. Kleppin 1991: 273). Die Quantität der mündlichen Fehlerkorrekturen im Bereich Grammatik richtet sich außerdem nach dem Fokus der Unterrichtsaktivität bzw. Aufgabe, die inhalts- oder aber formbasiert sein kann. So wird in inhaltsbasierten Sequenzen vermutlich nur begrenzt auf die grammatikalische Richtigkeit einer Äußerung geachtet werden, sodass die Anzahl der Fehlerkorrekturen in diesem Bereich abnehmen wird. Ferner ist zu vermuten, dass bei inhaltsorientierten Unterrichtsaktivitäten ein anderer Korrekturtyp zum Einsatz kommt als in formbasierten Einheiten.

Da der Fokus dieser Untersuchung auf dem Umgang der Lehrkraft mit fehlerhaften Schüleräußerungen im Bereich der prompts liegen soll, werden v.a. die Quantität und Qualität der verschiedenen Feedbackarten des Korrekturtyps II analysiert. Es wird daher einerseits zu ermitteln sein, welche Feedbackart die Lehrkraft innerhalb des Korrekturtyps mit negativer Evidenz am häufigsten verwendet, andererseits an wen das jeweilige Feedback wie adressiert wird. Feedbackarten, die zum Korrekturtyp II gehören, beinhalten stets eine Aufforderung zur Selbstkorrektur und damit einen Adressaten. Bei der Beobachtung des Lehrerfeedbacks soll also ebenfalls darauf geachtet werden, ob zu einer Eigen- oder Fremdkorrekturen aufgefordert wird, ob die Lehrkraft auf verbale oder non-verbale Korrekturaufforderungen zurückgreift und wie sie sich affektiv gegenüber den SchülerInnen und ihren mündlichen Fehlern verhält. Dabei ist die Menge der mündlichen Fehlerkorrekturen gepaart mit der affektiven Komponente in Bezug auf den Fehler allgemein, d.h. ob er grundsätzlich als etwas Positives oder Negatives angesehen wird, aber auch auf das negative Feedback für den Russischunterricht besonders relevant. Dies liegt an der im Vergleich zu anderen Fremdsprachen erhöhten Fehlerquote, die einerseits durch eine größere Distanz in der sprachlichen Verwandtschaft zwischen dem Deutschen und Russischen und daraus resultierend begrenzten lexikalischen Überschneidungen, andererseits durch die abweichende Schrift 
und komplexe russische Grammatik zu begründen ist (vgl. Lamnica 2015: $14 \mathrm{ff})$.

Schließlich soll der Versuch gewagt werden, den Erfolg der lehrerinitiierten Selbstkorrektur differenziert nach den einzelnen Feedbackarten mit negativer Evidenz festzustellen und nach dem Beispiel von Fagan eventuell Aussagen darüber zu treffen, ob die affektive Reaktion des Lehrers/der Lehrerin auf fehlerhafte Schüleräußerungen in Abhängigkeit zum Adressaten Auswirkungen auf den Erfolg der Selbstkorrektur hat.

Aus den vorangegangenen Überlegungen ergeben sich die folgenden Forschungsfragen bzw. Beobachtungsschwerpunkte:

1. Welchen Korrekturtyp setzt die Lehrkraft beim mündlichen Feedback im Bereich Grammatik häufiger ein?

2. Gibt es je nach Fokus der Unterrichtsaktivität, d.h. formbasiert oder inhaltsbasiert, Unterschiede bei der Wahl des Feedbacktyps?

3. Welche Feedbackart innerhalb des Korrekturtyps II verwendet die Lehrkraft am häufigsten?

4. Wer ist der Adressat des Feedbacks?

5. Wie wird dem Adressaten signalisiert, dass er eine Selbstkorrektur vornehmen soll?

6. Welche Botschaft, positive oder negative, wird durch das Korrekturverhalten der Lehrkraft den SchülerInnen bezüglich des Fehlers im Fremdsprachenunterricht auf sozialer Ebene vermittelt?

7. Ist die jeweils gewählte Art der Fehlerkorrektur erfolgreich, d.h. sind die SchülerInnen in der Lage sich selbst bzw. ihre MitschülerInnen zu korrigieren?

8. Hat die affektive Reaktion der Lehrkraft auf den gemachten Fehler Einfluss auf den Erfolg der Eigen- bzw. Fremdkorrektur?

Die Klassifizierung der Korrekturtypen bzw. Feedbackarten richtet sich nach der allgemein anerkannten Typologie von Lyster \& Ranta (1997). 


\section{Methodisches Vorgehen}

\subsection{TeilnehmerInnen}

Bei der beobachteten Lehrkraft handelt es sich um eine Russischlehrerin, die im Rahmen ihres Studiums Russisch als Fremdsprache gelernt hat und dieses Fach seit 5 Jahren an der Gesamtschule, an der diese Untersuchung durchgeführt wurde, unterrichtet. Sie legte ihr zweites Staatsexamen 2005 ab. Die beobachteten Korrektursequenzen fanden in den Unterrichtsstunden eines Russischkurses der 11. Jahrgangsstufe statt. Die insgesamt 11 SchülerInnen dieses Kurses lernen Russisch als Fremdsprache im vierten Lernjahr, wobei das sprachliche Niveau insgesamt sehr niedrig ist.

\subsection{Instrument}

Als Untersuchungsinstrument dient ein detaillierter Beobachtungsbogen, anhand dessen das Feedback der Lehrperson hinsichtlich der oben beschriebenen Gesichtspunkte kategorisiert werden kann. Dabei werden die Kategorien Feedbacktyp (mit positiver - negativer Evidenz), Vorgehen bei der Signalisierung des Fehlers (verbal - non-verbal), Feedbackart (recasts, explizite Korrektur, Klärungsaufforderung, Fehlerwiederholung, Elizitierung, metalinguistisches Feedback), der Adressat des Feedbacks (SchülerIn - MitschülerIn) sowie der Fokus der Aktivität (inhaltsbasiert - formbasiert) berücksichtigt. Die affektive Reaktion der Lehrkraft wird nach den Kategorien tadelnd, ironisch, freundlich, humorvoll und neutral bewertet, wobei die ersten beiden auf eine negative und die Restlichen auf eine positive bis neutrale Botschaft verweisen. Die Effektivität der Selbstkorrek- 
tur in Abhängigkeit der affektiven Lehrerreaktion wird in der Kommentarspalte in einem offenen Format festgehalten.

\subsection{Durchführung}

Es wurden insgesamt zehn Unterrichtsstunden zu je 45 Minuten in insgesamt acht Wochen beobachtet. Innerhalb dieser Beobachtungszeit war das Lehrerfeedback hauptsächlich auf Aussprachefehler und lexikalische Ungenauigkeiten gerichtet, sodass nur wenige Situationen beobachtet werden konnten, in denen tatsächlich mündliche Fehlerkorrektur auf grammatischer Ebene im Vordergrund stand. Dadurch zeichneten sich bereits zu Beginn der Untersuchung Probleme für eine quantitative Auswertung negativen Feedbacks im Bereich Grammatik ab, sodass der Fokus im Laufe der Durchführung verstärkt auf qualitative Gesichtspunkte verschoben wurde. Daraus ergab sich die Notwendigkeit, die einzelnen Korrektursequenzen sehr detailliert und möglichst wortgetreu zu dokumentieren, was seinerseits die Einschränkung der Beobachtungskapazitäten für andere Bereiche zur Folge hatte. Vor allem die Beobachtung der affektiven Reaktion der Lehrkraft auf die mündlichen Grammatikfehler stellte ein großes Problem dar, da es in den meisten Fällen nicht gelang, diese zeitgleich zur Aufzeichnung des Wortlauts zu beobachten bzw. zu dokumentieren. Somit müssen die Untersuchungsergebnisse auf einige wenige beobachtete Situationen beschränkt bleiben. 


\section{Ergebnisse}

Über einen Zeitraum von acht Wochen wurden 16 Unterrichtseinheiten (Doppel- sowie Einzelstunden) beobachtet, in denen der Beobachtungsbogen zehn Mal zum Einsatz kam. In den restlichen sechs Unterrichtseinheiten erfolgter nur wenig bis gar keine mündliche Fehlerkorrektur.

Insgesamt konnten zehn Korrektursequenzen mit positiver Evidenz und 17 Fehlerkorrekturen beobachtet werden, bei denen den SchülerInnen kein zielsprachliches Modell, sondern eine Aufforderung zur Eigen- bzw. Fremdkorrektur vorlag. Quantitativ überwog bei der beobachteten Lehrkraft demnach die Anwendung von Korrekturtyp II mit $63 \%$ gegenüber Typ I. Die quantitative Verteilung der einzelnen Feedbackarten ist der nachfolgenden Abbildung 1 zu entnehmen, wobei Fehlerwiederholung als Korrekturart kein einziges Mal zum Einsatz kam:
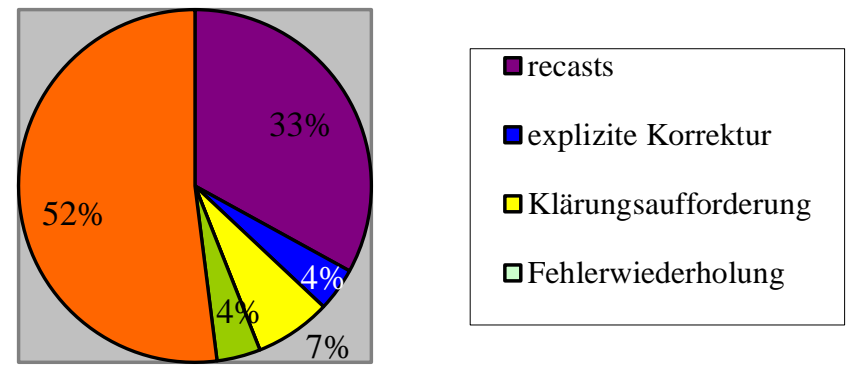

Abb. 1 Verteilung der angewendeten Feedbackarten (in \%)

Aufgrund der fokussierten Beobachtung von Grammatikfehlern haben nur wenige inhaltsbasierte Unterrichtssequenzen Eingang in die Auswertung erhalten. In den Fällen, in denen Grammatikfehler trotz des inhaltlichen Schwerpunktes korrigiert wurden, insgesamt neun an der Zahl, griff die Lehrkraft auf Korrekturarten mit positiver Evidenz - also den Korrekturtyp I - zurück (vgl. hierzu auch Sheen 2011), auch wenn sie manchmal 
nicht auf eine ergänzende metalinguistische Erklärung verzichten konnte. Der Einsatz von Korrekturtyp II konzentrierte sich hingegen auf den Bereich der formbasierten Aktivitäten (vgl. Abb. 2).
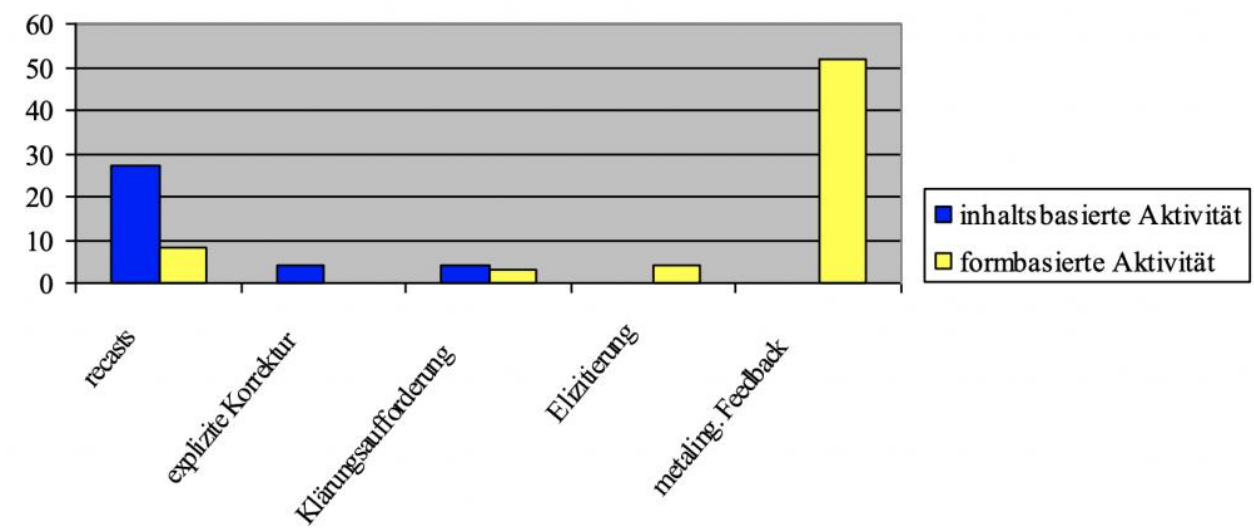

Abb. 2 Verteilung der Korrekturarten auf Aktivitätsschwerpunkte

Dabei konnte folgende Tendenz festgestellt werden, die hiernach anhand einiger Beispiele veranschaulicht werden sollen ${ }^{8}$. Bei Sequenzen, in denen es um die grammatische Korrektheit ging, forderte die Lehrerin fast ausschließlich zur Eigen- bzw. Fremdkorrektur auf, wenn sie davon ausgehen konnte, dass die SchülerInnen in der Lage sind, ihre Fehler selbst zu korrigieren. Dies war meistens dann der Fall, wenn es sich um grammatische Bereiche handelte, die Thema der aktuellen Unterrichtseinheit waren $^{9}$, wie im folgenden Beispiel:

Bsp. (19) S: Вы интересуетеся...

${ }^{8}$ Die Nummerierung folgt der im Anhang aufgeführten Auflistung aller Beispiele, die
nach Feedbackart sortiert sind.
${ }^{9}$ Eine einzige Ausnahme bildet das Beispiel 11, wobei man hier auch davon ausgehen
kann, dass die Lehrerin die Äußerung rein akustisch nicht ganz verstanden hat und den/
die SchülerIn daher zu einer Erklärung auffordern wollte.

Anastasia Drackert und Katrin Bente Karl (Hg.), Didaktik der slawischen Sprachen

Beiträge zum 2. Arbeitskreis in Innsbruck (19.02.-20.02.2018)

(C) 2019 innsbruck university press, ISBN 978-3-903187-80-1, DOI 10.15203/3187-80-1 
L: $\quad$ Guck mal, hier steht wieder ein Vokal am Ende.

Also kommt da wieder ein..?

S: $\quad$ 'C' und Weichheitszeichen...?

$\mathrm{L}$ : Ja, und wie wäre das dann?

S: $\quad$ Вы интересуетесь.

Wie Beispiel (20) zeigt, verfuhr die Lehrkraft auch ähnlich bei der Korrektur von Grammatikfehlern, die Teil einer (Wiederholungs-) Übung waren (vgl. auch die weiteren Bsp. 21-23 im Anhang) oder aber einer Leistungsüberprüfung angehörten (vgl. Bsp. 12 sowie die weiter unten zitierten Bsp. $13,16-17,24-26)$.

(20) S: ... чувствуете...

L: $\quad$ Nein, ist ja Singular nicht Plural.

S: $\quad$ Чувствуешь.

(12) S: Том умеет...

L: Und dann?

S: Игра.... ть?

L: Ja, genau!

Handelte es sich um Grammatikregeln, auf die die SchülerInnen hätten nicht sofort zugreifen können, weil diese nicht bekannt waren bzw. ihre Thematisierung im Unterricht zu weit zurücklag, formulierte die Lehrerin ihr Feedback so, dass die SchülerInnen keine Schwierigkeiten hatten, die entsprechende Regel abzurufen bzw. mithilfe des präzisen metalinguistischen Kommentars eine Selbstkorrektur durchzuführen, wie im Bsp. (14) (vgl. auch die weiter unten zitierten Bsp. 15, 18-19).

$\begin{array}{lll}\text { Bsp. (14) } & \text { L: } & \text { Was hieß denn noch mal 'klug'? } \\ & \text { S: } & \text { Умная. } \\ \text { L: } & \text { Und wir wollten das jetzt in maskulin aufschreiben, } \\ \text { also? } & \end{array}$




\section{S: Умный!}

Lag die Aktivität bzw. der Fehler jedoch außerhalb des eigentlichen Stundenschwerpunktes, griff die Lehrerin auch bei formorientierten Übungen durchaus auf recasts zurück, um die Aufmerksamkeit der SchülerInnen nicht vom wesentlichen Thema abzulenken, wie in den folgenden zwei Beispielen.
Bsp. (8) S: Они рисуят...
L: $\quad$ Они рисуют.
(9) S: Мы фотографирует...
L: $\quad$ Мы фотографируем.

Diese Beispiele stammen aus einer Stunde, in der der Fokus auf den neu eingeführten reflexiven Verben und nicht auf den Personalendungen bei der Konjugation von Verben mit dem Infix -ова-/-ева- lag. Diese Vorgehensweise wurde von der Lehrkraft jedoch nicht konsequent verfolgt, da sie in derselben Stunde auch auf metalinguistisches Feedback zurückgriff. Dieses war allerdings so detailliert, dass der Schüler auch bei einer Grammatikregel außerhalb des Stundenschwerpunkts erfolgreich eine Selbstkorrektur vornehmen konnte.
Bsp. (18) S: Я интересуюсь в футбол.
L: $\quad$ Ja, und jetzt lässt du das 'B' weg und setzt das
'футбол' in den Instrumental.
S: Футболом?
L: Ja, genau!

In inhaltsbasierten Sequenzen hingegen versuchte die Lehrkraft, ihre SchülerInnen grammatikalisch zu entlasten und verwendete unabhängig davon, ob es sich um neu gelernte grammatische Elemente handelt oder nicht, verstärkt Korrekturtyp I. Dabei zeigt Beispiel (5) eine Korrektur aus einer Stunde, die unmittelbar der Unterrichtseinheit folgte, in der die Konjugation der -ова-/-ева-Verben besprochen wurde, d.h. eine für die SchülerInnen neue grammatische Regel. 


$\begin{array}{llll}\text { Bsp. (5) } & \text { S: } & \text { Интересеу... (unverständlich) } \\ & \text { L: } & \text { Интересуешься. } \\ & \text { (6) } & \text { S: } & \text { Я играю на флейта. } \\ & \text { L: } & \text { На флейте. }\end{array}$

Auffällig ist, dass Korrekturtyp II, der sich zu 82\% aus metalinguistischem Feedback zusammensetzte, hauptsächlich in den Unterrichtsstunden zum Einsatz kam, in denen überwiegend grammatische Themen behandelt wurden. Nur in zwei Fällen (vgl. Bsp. 14 und 15) verwendete die Lehrkraft diese Feedbackart in Stunden, in denen das freie Sprechen bzw. die Lexik im Vordergrund standen. So machte sie beispielsweise einen Schüler, der sich bei seiner Aussage an der englischen bzw. deutschen Konstruktion zu orientieren begann, darauf aufmerksam, dass im Russischen die entsprechende Form des Verbs sein im Präsens wegfällt:

Bsp. (15) S: Marco is большой.

L: $\quad$ Nein, das ist Englisch. Im Russischen fällt das

'sein' im Präsens weg.

S: $\quad$ Also nur 'Марко большой'?

L: Genau.

In der Regel griff die Lehrerin in solchen Unterrichtsstunden auf Feedback mit positiver Evidenz zurück, da der Inhalt der Schüleraussagen bzw. das freie Sprechen an sich im Fokus standen und der Gesprächsfluss somit einigermaßen aufrechterhalten werden konnte. In den Situationen, in denen der gemachte Fehler nach Einschätzung der Lehrerin jedoch eine grundsätzliche bzw. für das weitere Unterrichtsgespräch relevante Grammatikregel verletzte, weil sie beispielsweise davon ausgehen konnte, dass die gewählte Form im weiteren Verlauf der Stunde noch häufig auftreten würde, fügte sie ihrem recast bzw. der expliziten Korrektur einen metalinguistischen Kommentar hinzu, um der Bedeutung der Regel einen gewissen Nachdruck zu verleihen:

Bsp. (3) S: Он нравится делать...

L: $\quad$ 'Ему нравится делать', weil es Dativ ist. 


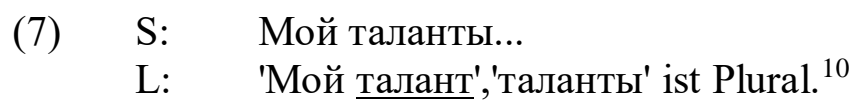

Im Gegensatz zu den Untersuchungsergebnissen von Fagan (2015) äußerte die beobachtete Lehrkraft nur in einigen wenigen Situationen, in denen sie die SchülerInnen zur Selbstkorrektur aufforderte, positives Feedback. Zwar erhielten die SchülerInnen in neun von 17 Korrektursequenzen eine Bestätigung, dass sie den gemachten Fehler richtig korrigiert hatten, jedoch reagierte die Lehrerin in weniger als der Hälfte der Fälle mit einem tatsächlichen Lob. Dieses hob sich lediglich durch eine etwas gehobenere bzw. enthusiastische Stimm(lag)e von den sonstigen Bestätigungen ab.

Bsp. (25) S: Фотографируетеся.

L: Wo nimmst du denn das '-сь' her?

(S murmelt etwas Unverständliches)

L: Du orientierst dich jetzt an dem, was an der Tafel steht und das ist ein cя-Verb.

S: Dann 'фотографируешь'.

L: Genau.

Der Wortlaut des positiven Feedbacks nach der Selbstkorrektur beschränkte sich insgesamt auf ,ja“, ,richtig“ und ,genau“ (vgl. Bsp. 11, 12, 13, 15, 17, 18, 23, 25), wobei das Beispiel 26 verdeutlicht, dass eine

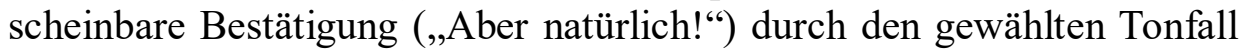
als Vorwurf interpretiert werden kann. Zum Teil wurde die Selbstkorrektur gar nicht kommentiert (vgl. Bsp. 14, 16, 19-22, 24), obwohl es sich um Unterrichtsaktivitäten handelte, bei denen die Form und nicht der Inhalt im Vordergrund stand. In die gleiche Richtung deuten auch die Befunde von Kleppin \& Königs (1991: 285 f).

\footnotetext{
${ }^{10}$ Eine Ausnahme bildet das Beispiel 7, in dem die Lehrerin die SchülerInnen über die Pluralform der Vokabel талант aufklärt, die auch im weiteren Gesprächsverlauf von Bedeutung ist (s. Anhang).
} 
Neben dem spärlich gesäten positiven Feedback beschränkten sich die Beobachtungen auf dem Gebiet der affektiven Reaktion der Lehrerin auf wenige Situationen, in denen negativ besetzte Mimik, wie Stirnrunzeln (Bsp. 24), Augenrollen (Bsp. 16) und ein vorwurfsvoller Gesichtsausdruck (Bsp. 26) registriert werden konnten. Verstärkt wurde diese negative Botschaft zum Teil durch einen tadelnden Tonfall der Lehrerin, erhöhte Lautstärke sowie ein direktes Ansprechen der Schülerin, die den Fehler gemacht hatte, mit Namen:

Bsp.
(16) S: Он играть...
L: Sarah! (rollt mit den Augen; vorwurfsvoll) Das ist der Infinitiv.
S: Играет.
(26) S: Играть на гитара...?
L: Ha! (macht ein vorwurfsvolles Gesicht)
S: $\quad$ Гитаре?
L: (vorwurfsvoll) Aber natürlich!

Der Einsatz non-verbaler Fehlersignale erfolgte insgesamt sehr selten und konnte nur in Kombination mit anderen Feedbackarten, wie Elizitierung, beobachtet werden.

\begin{tabular}{|c|c|c|}
\hline Bsp. (13) & $\begin{array}{l}\text { S: } \\
\text { L: } \\
\text { S: } \\
\text { L: }\end{array}$ & $\begin{array}{l}\text { Тренирут... } \\
\text { Я - я - я! (zeigt an die Tafel) } \\
\text { Тренируюсь? } \\
\text { Ja! }\end{array}$ \\
\hline
\end{tabular}

Anders als bei Fagan (2015) erfolgte kaum eine Differenzierung in der Adressierung negativen Feedbacks. In 88\% der Fälle, in denen zur Selbstkorrektur aufgefordert wurde, richtete sich das Feedback an den/die SchülerIn, der/die den Fehler gemacht hatte. Auch die anschließende Korrektur, die in 16 von 17 Fällen erfolgreich war, erfolgte durch den Urheber des Fehlers. Lediglich zwei Mal kam es zu einer Fremdkorrektur (vgl. Bsp. 17 und 24). Beide Male handelte es sich jedoch um Situationen, bei denen die Eigenkorrektur gescheitert war, sodass das negative Feedback sich auch 
hier zunächst an den Urheber des Fehlers richtete:

$\begin{array}{ll}\text { Bsp. (17) } & \mathrm{S}_{1}: \quad \text { Я интересую... } \\ & \mathrm{L}: \quad \text { Die 1. Person Singular von 'интересоваться'? } \\ \text { (guckt zuerst } S_{1} \text {, dann alle anderen an) } \\ \mathrm{S}_{2}: \quad \text { Интересуюсь. } \\ \mathrm{L}: \quad \text { Richtig. } \\ \text { (24) } \mathrm{S}_{1}: \quad \text { Интересуетесь. } \\ \mathrm{L}: \quad \text { Nein, das wäre die 2. Person Plural. } \\ \\ \mathrm{S}_{1}: \quad \text { Интересуемем... (Endung unverständlich) } \\ \text { (L runzelt die Stirn) } \\ \mathrm{S}_{2}: \quad \text { Интересуемся. }\end{array}$

\section{Diskussion}

Die Untersuchung hat gezeigt, dass insgesamt nur wenig mündliche Korrektur im Bereich Grammatik stattfindet. Zugleich muss festgehalten werden, dass, ungeachtet der Tatsache, dass sich die SchülerInnen offiziell im vierten Lernjahr befinden, sie über ein sehr niedriges Sprachniveau verfügen. Den Erkenntnissen von Kleppin \& Königs (vgl. 1991: 273) zufolge müsste zwar gerade hier ein hoher Korrekturanteil zu beobachten sein, doch verzichtet die Lehrerin auf die Korrektur zahlreicher basaler Fehler im Unterrichtsgespräch. Zu begründen wäre diese Beobachtung dadurch, dass die SchülerInnen kaum in vollständigen Sätzen sprechen und der Lehrperson somit nur wenig Gelegenheit zur Korrektur bleibt. Allerdings ist auch nicht auszuschließen, dass es sich um das individuelle Korrekturverhalten der Lehrerin handelt.

Korrigierte sie doch, so bevorzugte sie Korrekturtyp I. Zu erklären wäre dieses Verhalten mit der Absicht der Lehrerin, die SchülerInnen nicht durch zu viele Korrekturen im mündlichen Sprachgebrauch zu entmutigen 
und ihre Aufmerksamkeit v.a. in konversationslastigen Unterrichtseinheiten nicht auf - in diesen Fällen - nebensächliche grammatische Phänomene zu lenken. Hier griff sie daher eher auf ein explizit korrektives, modelllieferndes Feedback mit positiver Evidenz zurück. Dadurch gelang es ihr einerseits, die Übermittlung von Informationen nicht zu behindern, und andererseits die Sprechkompetenz ihrer SchülerInnen intensiver zu fördern. Bedingt durch ihr Korrekturverhalten, bei dem sie die gemachten Fehler im Verlauf des Gesprächs direkt verbesserte und kaum auf explizite Korrekturen zurückgriff, konnte die Konversation trotz der gemachten Fehler ungehindert und schnell fortgeführt werden.

Diese Vorgehensweise erscheint plausibel, betrachtet man sie vor dem Hintergrund der vergleichsweise schwer zu erlernenden russischen Sprache, des Fachs Russisch und seiner meist nachrangigen Stellung im Fächerkanon sowie der fehlenden Relevanz des Faches für das Abitur an der Schule, an der die Untersuchung durchgeführt wurde. Die Aufrechterhaltung der Motivation wird in diesem konkreten Fall also höher gewichtet als sprachliche Korrektheit, was sich in den inhaltsbasierten Unterrichtssequenzen zwangsläufig auf das Korrekturverhalten der Lehrerin auswirkte. Einige wenige Ausnahmen bildeten die Korrektursequenzen, in denen die Lehrkraft trotz des eindeutigen inhaltsbasierten Schwerpunktes das Feedback mit positiver Evidenz um metalinguistische Kommentare ergänzte. Es lässt sich vermuten, dass diese Interventionen in Kauf genommen wurden, um die Aufmerksamkeit der SchülerInnen auf solche grammatischen Phänomene zu lenken, deren Verwendung von der Lehrerin auch im weiteren Unterrichtsverlauf geplant bzw. antizipiert wurde.

Trotzdem wurden die SchülerInnen in den Unterrichtsphasen, in denen die Einführung bzw. konkrete Thematisierung bestimmter grammatischer Phänomene und Regeln im Mittelpunkt stand, d.h. bei grundsätzlich formbasierten Aktivitäten, überwiegend zur Eigenkorrektur aufgefordert. Dabei ließ die Lehrerin den SchülerInnen viel Zeit für die Verbesserung ihres Fehlers, die nur dann von einem/einer MitschülerIn übernommen wurde, 
wenn die Eigenkorrektur nicht gelang. Das Adressieren des negativen Feedbacks an den Urheber des Fehlers zeichnet ein völlig anderes Bild als in der Untersuchung von Fagan (2015: 82), bei der die beobachtete Lehrkraft die Aufmerksamkeit aktiv vom Fehlerproduzenten ablenkte und die Korrektur entweder selbst vornahm oder die anderen SchülerInnen durch prompts dazu aufforderte. Das Verhalten der Lehrperson in der gegenwärtigen Untersuchung stützt eher die Beobachtungen von Kleppin \& Königs (1991: 275), die ebenfalls eine verstärkte Zuwendung zum Urheber des Fehlers beobachteten. Die Initiierung der Korrektur erfolgte stets verbal. Die wenigen non-verbalen Signale dienten eher als Verstärkung bzw. unterstrichen die affektive Reaktion der Lehrerin. Das Fehlen gruppeninterner non-verbaler Signale, die laut Kleppin (1997: 97) das Potential besitzen, wenig in den Redefluss des Schülers/der Schülerin einzugreifen und gleichzeitig eine günstige und angstfreie Gruppenatmosphäre zu fördern, kann darauf zurückgeführt werden, dass die Lehrerin besagte Lerngruppe erst vor Kurzem übernommen hatte und sich solche Routinen noch nicht etabliert haben.

Durch den Einsatz des Korrekturtyps II, der vorrangig aus metalinguistischem Feedback bestand, wurden die SchülerInnen dazu veranlasst die entsprechende Grammatikform selbstständig zu bilden. Gleichzeitig hatten sie die Möglichkeit das konkrete Phänomen zu reflektieren und dadurch Sprachbewusstsein aufzubauen. Der bevorzugte Gebrauch dieser Feedbackart entspricht den aktuellen Tendenzen in der Fremdsprachendidaktik, die auf positive Einflüsse eines ausgebildeten Sprachbewusstseins für den Fremdsprachenerwerb setzen (vgl. Schoormann \& Schlak 2011: 48 f; Tönshoff 2005: 9 ff).

Dieses Vorgehen setzte die Lehrkraft bei bereits früher erlernten, aber einfachen und häufig gebrauchten grammatischen Phänomenen ein. Die limitierten Grammatikkenntnisse der SchülerInnen ließen sie jedoch davon absehen, diese Feedbackstrategie bei grammatischen Regeln mit einer nied- 
rigeren Frequenz im Sprachgebrauch des Fremdsprachenunterrichts anzuwenden. Ausnahmen bildeten ebenfalls die Fälle, in denen der Fokus auf einem anderen grammatischen Bereich als dem fehlerhaften lag. Dadurch, dass von den sieben analysierten Unterrichtseinheiten vier auf Grammatik ausgerichtet waren, konnten insgesamt mehr Korrektursequenzen mit negativer Evidenz beobachtet werden. Dieses Ergebnis steht in einem deutlichen Gegensatz zu den Beobachtungen von Kleppin \& Königs (vgl. 1991: $274 \mathrm{ff}$ ), die von einer überwiegend von der Lehrkraft ausgehenden Korrektur mit positvier Evidenz im Bereich der Morphosyntax berichten. Allerdings könnten die gemachten Beobachtungen auch durch die langsam fortschreitenden Veränderungen im Fremdsprachunterricht hin zu einer schülerorientierten bewusstheitsfördernden Ausrichtung in den vergangenen Jahrzehnten erklärt werden. Eine ,sich selbst entwickelnde Einsicht“ (ebd. 1991: 283) in die Sprache seitens der SchülerInnen scheint nun zumindest bei der beobachteten Lehrperson - Priorität zu haben.

Die Beobachtung der affektiven Reaktion der Lehrerin gestaltete sich äußerst schwierig, sodass die Bilanz nur vorsichtig formuliert werden darf. Affektive Reaktionen auf fehlerhafte Schüleräußerungen waren häufig kritischer Natur. Meist signalisierte die Lehrerin ihre Unzufriedenheit mimisch oder durch tadelnde Ausrufe. Positive Reaktionen in Bezug auf Fehler konnten leider kaum beobachtet werden. Sie folgten eher einer erfolgreichen Korrektur. Da positive Emotionen aber häufig durch unscheinbare und flüchtige Gestik bzw. Mimik ausgedrückt werden, könnte die geringe Anzahl positiver Lehrerreaktionen auf Schülerfehler durchaus als ein Resultat der begrenzten Beobachtungsmöglichkeiten betrachtet werden. Grundsätzlich wäre die Unterstützung durch Audio- bzw. Videoaufnahmen erforderlich gewesen, um affektive Reaktionen besser erfassen und den zuvor gesetzten Schwerpunkt, d.h. eine qualitative Analyse des Lehrerfeedbacks im Bereich der prompts, während der Untersuchung besser verfolgen zu können. 
Allerdings legt gerade die Tatsache, dass positives Feedback in Korrektursituationen nur wenig Raum einnimmt bzw. unscheinbar ausfällt, die Vermutung nahe, dass der Fehler weiterhin sowohl in den Schülerköpfen als auch in der Vorstellung der beobachteten Lehrerin negativ konnotiert zu sein scheint. Die Fehlerkultur, auf deren Wandel in der fachdidaktischen Literatur viel Wert gelegt wird, scheint sich daher kaum verändert zu haben. Dies müsste jedoch erst durch weitere Untersuchungen nachgewiesen werden. In einem auffallenden Gegensatz dazu stehen sowohl die Beobachtungen von Kleppin \& Königs (vgl. 1991: 280), wie auch die von Fagan (vgl. 2015: 78 ff). Beide Studien berichten von einer eher positiven Einstellung der Lehrenden zum Phänomen des Fehlers im Fremdsprachenunterricht.

Die Beantwortung der letzten Forschungsfrage nach dem Zusammenhang zwischen der affektiven Lehrerreaktion und dem Erfolg der Selbstkorrektur gestaltet sich aufgrund der mangelhaften Datenlage als besonders schwierig. Die affektive Reaktion der Lehrkraft scheint zwar keinen Einfluss auf den unmittelbaren Korrekturerfolg zu haben, allerdings könnte aufgrund des wiederholten Auftretens ein und desselben Fehlers (vgl. die Konjugation des Verbs интересоваться) vermutet werden, dass die in diesem Zusammenhang von der Lehrerin vermittelten, überwiegend negativen Emotionen gegenüber den SchülerInnen möglicherweise den Lernerfolg hemmen. Allerdings dürfen auch Faktoren wie der Einfluss der L1 auf die zu erlernden L2, die psycholinguistischen Voraussetzungen der Lerner, d.h. die Entwicklungsstufe der Interimsprache, auf die die Komplexität des zu vermittelnden Sprachphänomens abgestimmt sein muss ${ }^{11}$, sowie weitere soziolinguistische Faktoren, z.B. Lernerpersönlichkeit, phonetische Begabung, Alter u.a., nicht außer Acht gelassen werden (vgl. Schlak 2003: 4 ff). Auch sie beeinflussen die mentalen Sprachverarbeitungsprozesse und den Lernerfolg, konnten im Rahmen der gegenwärtigen Studie aber nicht weiter untersucht werden.

${ }^{11}$ Vgl. hierzu die ,teachability“-Hpothese nach Pienemann (Schlak 2004: 60 ff).

Anastasia Drackert und Katrin Bente Karl (Hg.), Didaktik der slawischen Sprachen

Beiträge zum 2. Arbeitskreis in Innsbruck (19.02.-20.02.2018)

(C) 2019 innsbruck university press, ISBN 978-3-903187-80-1, DOI 10.15203/3187-80-1 
Sämtliche Vermutungen über die Gründe für das jeweilige Korrekturverhalten der Lehrkraft resultieren allein aus der Interpretation des beobachteten Verhaltens. Für eine Triangulation dieser Beobachtungsergebnisse wäre ein eingehendes Interview mit der Lehrkraft sowie die Befragung der SchülerInnen nötig, um die Beweggründe hinter den einzelnen Korrekturhandlungen tatsächlich als Motive bezeichnen und die affektive Wirkung der Fehlerkorrektur auf die SchülerInnen ermitteln zu können. Dies nimmt die Autorin durch die Erweiterung der Studie in ihrer Masterarbeit vor (Feldmann 2017).

Insgesamt fällt es bei der geringen Menge an Datenmaterial schwer das Verhalten der Lehrerin zu verallgemeinern und daraus Handlungsempfehlungen für den Unterricht abzuleiten. Die meisten Korrektursituationen bleiben Einzelfälle und geben damit nur einen kleinen Einblick in die Möglichkeiten der mündlichen Fehlerkorrektur im Russischunterricht, die immer von einer großen Anzahl anderer Einflussfaktoren abhängig sind. Nichtsdestotrotz können die gewonnenen Erkenntnisse zu einer Reflexion des eigenen Korrekturverhaltens beitragen, den Umgang mit Schülerfehlern verbessern und dadurch das darin liegende Potenzial bei der Entwicklung der Sprachkenntnisse weiter ausschöpfen.

\section{Literatur}

Cathcart, R.L. \& Olsen, J.E.W. (1976). Teachers' and Students' Preferences for Correction of Classroom Conversation Errors. In J.F. Fanselow \& R. Crymes (Hrsg.), On TESOL 1976 (41-53). Washington D.C.: TESOL.

Demme, S. (2001). “...über Fehler beim Fremdsprachenlernen sollten wir noch viel mehr erfahren." - Wie DaF-Studierende ihre Erfahrungen im Umgang mit Fehlern beim Fremdsprachenlernen reflektieren. In 
K. Aguado \& C. Riemer (Hrsg.), Wege und Ziele. Theorie, Empirie und Praxis des Deutschen als Fremdsprache (und anderer Fremdsprachen). Festschrift für Gert Henrici zum 60. Geburtstag (227244). Baltsmannsweile: Schneider Verlag Hohengehren.

Ellis, R., Basturkmen, H. \& Loewen, S. (2001). Learner uptake in communicative ESL lessons. Language Learning 51, 281-318.

Fagan, D.S. (2015). Managing language errors in real-time: A microanalysis of teacher practices. System 55, 74-85.

Feldmann, B. (2017). Wie nehmen Schüler die Korrektur ihrer Lehrer wahr? Eine empirische Untersuchung zur affektiv-emotionalen Dimension mündlicher Fehlerkorrekturen im Russischunterricht. Bochum. (nicht veröffentlichte Masterarbeit)

Gnutzmann, C. \& Kiffe, M. (1993). Mündliche Fehler und Fehlerkorrekturen im Hochschulbereich. Zur Einstellung von Studierenden der Anglistik. Fremdsprachen lehren und lernen 22, 91-108.

Havranek, G. (2002). Die Rolle der Korrektur beim Fremdsprachenlernen. Frankfurt a.M.: Peter Lang.

Kim, J. \& Han, Z. (2007). Recasts in communicative EFl classes: Do teacher intent and learner interpretation overlap? In A. Mackey (Hrsg.), Conversational interaction in second language acquisition: A series of empirical studies (269-297). Oxford: Oxford University Press.

Kleppin, K. (1997). Fehler und Fehlerkorrektur. Berlin u.a.: Langenscheidt.

Kleppin, K. \& Königs, F.G. (1991). Der Korrektur auf der Spur. Untersuchungen zum mündlichen Korrekturverhalten von Fremdsprachenlehrern. Bochum: Brockmeyer.

Lamnica, A. (2015). Praktikumsbericht Orientierungspraktikum. RuhrUniversität Bochum.

Lochtmann, K. (2002). Korrekturhandlungen im Fremdsprachenunterricht. Bochum: AKS Verlag.

Nystrom, N.J. (1983). Teacher-student interaction in bilingual classroom: Four approaches to error feedback. In H.W. Seliger \& M.H. Long

Anastasia Drackert und Katrin Bente Karl (Hg.), Didaktik der slawischen Sprachen

Beiträge zum 2. Arbeitskreis in Innsbruck (19.02.-20.02.2018)

(C) 2019 innsbruck university press, ISBN 978-3-903187-80-1, DOI 10.15203/3187-80-1 
(Hrsg.), Classroom Orientated Research in Second Language Acquisition (169-189). Rowley: Newbury House.

Mehlhorn, G. (i.Dr.). Russischdidaktik State of the Art.

Forschungsüberblick 2008-2018. Fremdsprachen lernen und lehren. FLuL.

Ministerium für Schule und Weiterbildung des Landes Nordrhein-Westfalen (2014) (Hrsg.), Kernlehrplan für die Sekundarstufe II Gymnasium/Gesamtschule in Nordrhein-Westfalen. Russisch. Düsseldorf. Verfügbar unter http://www.schulentwicklung.nrw.de/lehrplaene/ upload/klp_SII/r/KLPGOSt_Russisch.pdf [18.02.2017].

Schoormann, M. \& Schlack, T. (2011). Hilfreich oder ohne praktischen Nutzen? - Die Forschung zur mündlichen Fehlerkorrektur im Zweitund Fremdsprachenunterricht. Zeitschrift für Fremdsprachenforschung 22, 1, 43-84.

Schlak. T. (2003). Die Auswahl grammatischer Lernziele: Linguistische, psycholinguistische und didaktische Perspektiven. Zeitschrift für Interkulturellen Fremdsprachenunterricht, 8,1. Verfügbar unter https://zif.spz.tu-darmstadt.de/jg-08-1/beitrag/schlak4.htm [07.07.2019].

Schlak, T. (2004). Zur Rolle expliziter Grammatikvermittlung im universitären DaF-Unterricht: Eine qualitativ-ethnographische Fallstudie. German as a Foreign Language 2, 40-80.

Schulz, R.A. (2001). Cultural Differences in student and teacher perceptions concerning the role of grammar instruction and corrective feedback. The Modern Language Journal 85, 244-258.

Sheen, Y. (2011). Corrective Feedback, Individual Differences and Second Language Learning. Dortrecht u.a.: Springer.

Stadler, W. (2014). Der Fehler im Russischunterricht. In A. Bergmann (Hrsg.), Fachdidaktik Russisch (282-283). Tübingen: Narr.

Tönshoff, W. (2005). Mündliche Fehlerkorrektur im Fremdsprachenunterricht. Ein Blick auf neuere empirische Untersuchungen. Zeitschrift für Fremdsprachenforschung 16, 1, 3-21. 


\section{Anhang}

\section{Auswertungstabelle Feedbackarten}

\begin{tabular}{|c|c|c|c|}
\hline Nr. & Feedbackart & Fokus der Aktivität & Stundenthema \\
\hline & Recasts & inhaltsbasiert & \\
\hline 1 & & $\begin{array}{l}\mathrm{S}: \quad \text { Он } \quad \text { не } \quad \text { молодый... } \\
\text { молодай...? } \\
\mathrm{L}: \quad \text { Он не молодой. }\end{array}$ & Lexik \\
\hline 2 & & $\begin{array}{l}\text { Он не молодае... } \\
\text { Он не молодой. }\end{array}$ & Lexik \\
\hline 3 & $\begin{array}{l}+\quad \text { metaling. } \\
\text { Feedback }\end{array}$ & $\begin{array}{l}\text { S: } \text { Он нравится делать... } \\
\text { L: 'Eмy нравится делать', } \\
\text { weil es Dativ ist. }\end{array}$ & $\begin{array}{l}\text { Personenbeschrei- } \\
\text { bung/Charakterisie- } \\
\text { rung }\end{array}$ \\
\hline 4 & & $\begin{array}{l}\text { Я хорошо плавать. } \\
\text { Я хорошо плаваю. }\end{array}$ & Charakterisierung \\
\hline 5 & & $\begin{array}{l}\text { S: } \quad \text { Интересеу... } \\
\text { (unverständlich) } \\
\text { L: } \quad \text { Интересуешься. }\end{array}$ & Charakterisierung \\
\hline 6 & & $\begin{array}{l}\text { Я играю на флейта. } \\
\text { На флейте. }\end{array}$ & Charakterisierung \\
\hline 7 & $\begin{array}{l}+\quad \text { metaling. } \\
\text { Feedback }\end{array}$ & 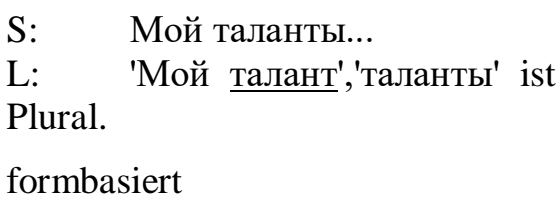 & Charakterisierung \\
\hline 8 & & $\begin{array}{l}\text { Они рисуят... } \\
\text { Они рисуют. }\end{array}$ & Reflexive Verben \\
\hline 9 & & $\begin{array}{l}\text { Мы фотографирует... } \\
\text { Мы фотографируем. }\end{array}$ & Reflexive Verben \\
\hline
\end{tabular}




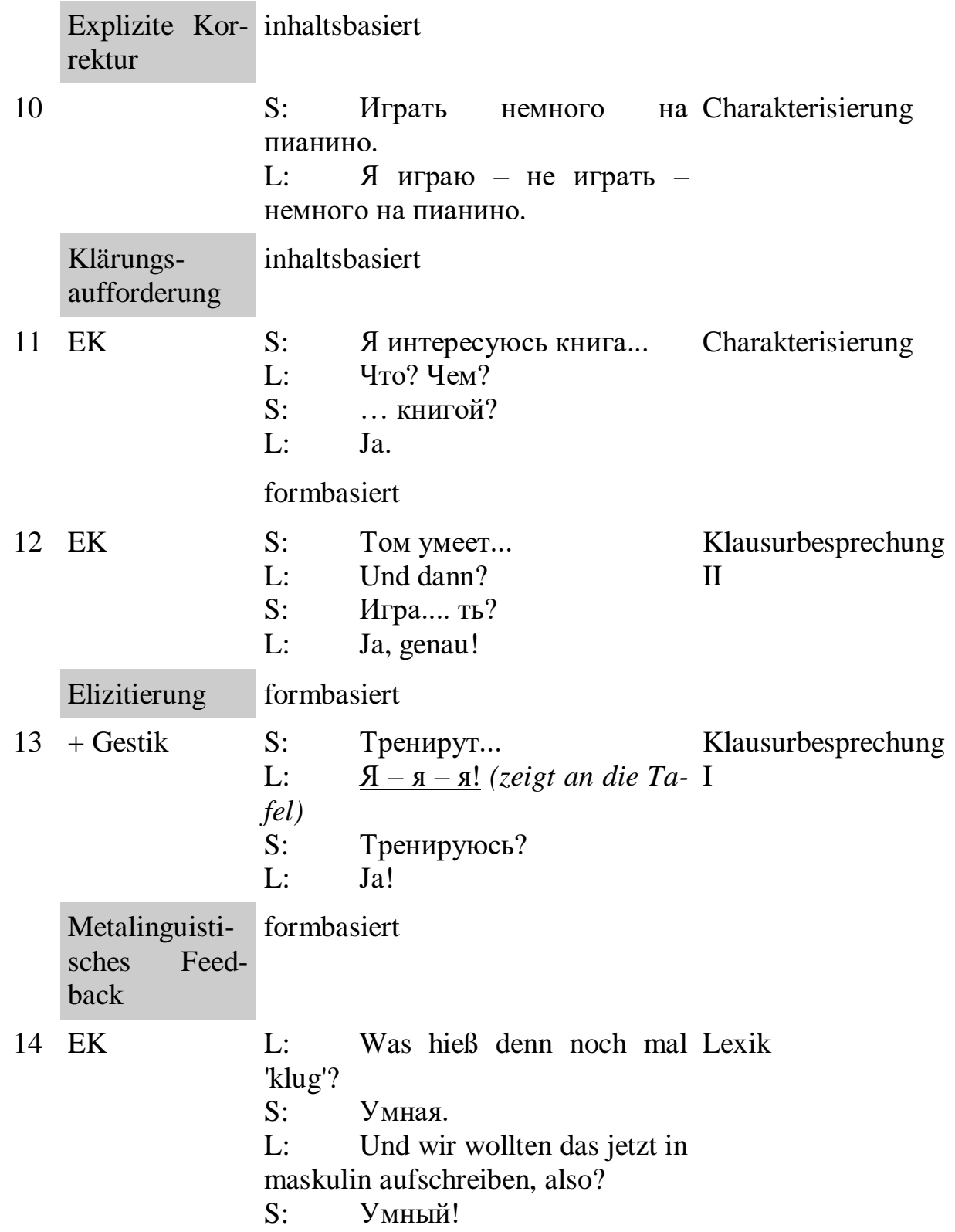

12 EK S: Том умеет...

L: Und dann? II

S: Игра.... ть?

L: Ja, genau!

Elizitierung formbasiert

13 + Gestik S: Tренирут... Klausurbesprechung

L: $\quad \underline{\text { G }-\mathrm{A}-\mathrm{g} !}$ (zeigt an die Ta- I

fel)

S: Tренируюсь?

L: Ja!

Metalinguisti- formbasiert sches Feedback

14 EK L: Was hieß denn noch mal Lexik 'klug'?

S: Умная.

L: $\quad$ Und wir wollten das jetzt in maskulin aufschreiben, also?

S: Умный! 
15 EK

17 FK

18 EK

19 EK

EK
$\mathrm{S}: \quad$ Marco is большой.

Personenbeschrei-

L: Nein, das ist Englisch. Im bung/CharakterisieRussischen fällt das 'sein' im Prä- rung sens weg.

S: Also nur 'Джастин

большой'?

L: Genau.

$16+$ Mimik

S: Он играть...

L: $\quad$ Sarah! (rollt mit den Augen) II

Das ist der Infinitiv.
$\rightarrow$ tadelnd
S: Играет.

$\mathrm{S}_{1}:$ Я интересую...

L: Die 1. Person Singular von II

'интересоваться'?

(guckt zuerst $S_{1}$, dann alle

anderen an)

$\mathrm{S}_{2}: \quad$ Интересуюсь.

L: Richtig.

S: $\quad$ Я интересуюсь в футбол. Reflexive Verben

L: $\quad$ Ja, und jetzt lässt du das 'B'

weg und setzt das 'футбол' in den

Instrumental.

S: $\quad$ Футболом?

L: Ja, genau!

S: $\quad$ Вы интересуетеся... Reflexive Verben

L: $\quad$ Guck mal, hier steht wieder

ein Vokal am Ende. Also kommt da wieder ein...?

S: 'C' und Weichheitszeichen...?

L: $\quad$ Ja, und wie wäre das dann?

S: $\quad$ Вы интересуетесь. 
20 EK

S: $\quad$... чувствуете...

Grammatikübung zur

L: Nein, ist ja Singular nicht Konjugation der VerPlural.

ben auf -ова-/-ева-

S: $\quad$ Чувствуешь.

21 EK

S: $\quad$... паникоть...?

Grammatikübung zur

L: Ne, hier kommt die 3. Per- Konjugation der Verson Singular hin.

ben auf -ова-/-ева-

S: Паникует.

22 EK

S: $\quad$... шокирует.

L: 2. Person Plural?

S: Шокируете.

Grammatikübung zur Konjugation der Verben auf -ова-/-ева-

23 EK

S: Програмируют.

Grammatikübung zur

L: $\quad$ 1. Person Singular ist...?

S: Програмирую.

Konjugation der Ver-

L: Genau!

ben auf -ова-/-ева-

$24+$ Mimik

$\mathrm{S}_{1}$ : Интересуетесь.

FK

L: Nein, das wäre die 2. Person I

Plural.

$\mathrm{S}_{1}$ : Интересуемем... (Endung

unverständlich)

(L runzelt die Stirn) $\rightarrow$ unzufrieden

$\mathrm{S}_{2}$ : Интересуемся.

25 EK

S: $\quad$ Фотографируетеся. Klausurbesprechung

L: Wo nimmst du denn das '- I

cь' her?

(S murmelt etwas Unverständliches)

L: $\quad$ Du orientierst dich jetzt an

dem, was an der Tafel steht und das

ist ein ся-Verb.

S: Dann 'фотографируешь'.

L: Genau.

Mimik

formbasiert 
$26+$ metaling. S: Играть на гитара...? Klausurbesprechung Feedback bzw. L: Ha! (macht ein vorwurfs- I Elizitierung volles Gesicht $) \rightarrow$ tadelnd EK S: Гитаре?

L: (vorwurfsvoll) Aber natürlich!

Gestik formbasiert

27 + Recast S: Я интересуем... Reflexive Verben

L: $\quad$ (zeigt an die Tafel, wo die richtige Konjugationsform steht; $S$ reagiert nicht) Интересуюсь! 
